\title{
Abate humanitário de aves: Revisão
}

\section{Denise Russi Rodrigues ${ }^{1 *}$, Fabiana Ramos dos Santos ${ }^{2}$, Weslane Justina da Silva ${ }^{3}$, Alison Batista Vieira Silva Gouveia ${ }^{4}$, Cibele Silva Minafra ${ }^{5}$}

\author{
${ }^{1}$ Doutoranda em Ciência Animal-Universidade Federal de Goiás- UFG, Goiânia, Goiás, Brasil E-mail: \\ veterinariadenise@hotmail.com \\ ${ }^{2}$ Docente Instituto Federal Goiano - Campus Rio Verde, Rodovia Sul Goiana, Km 01, Zona Rural, Caixa Postal 66, CEP - \\ 75.901-970, Rio Verde, Goiás, Brasil.E-mail: fabiana.santos@ifgoiano.edu.br \\ ${ }^{3}$ Pós-Graduação Mestrado em Zootecnia do Instituto Federal Goiano, Campus Rio Verde, Rodovia Sul Goiana, Km 01, Zona \\ Rural, Caixa Postal 66, CEP - 75.901-970, Rio Verde, Goiás, Brasil.E-mail: weslanejds@gmail.com \\ ${ }^{4}$ Graduando em Zootecnia do Instituto Federal Goiano, Campus Rio Verde, Rodovia Sul Goiana, Km 01, Zona Rural, Caixa \\ Postal 66, CEP-75.901-970, Rio Verde, Goiás, Brasil.E-mail: alisonmestre@ hotmail.com \\ ${ }^{5}$ Docente Instituto Federal Goiano - Campus Rio Verde, Rodovia Sul Goiana, Km 01, Zona Rural, Caixa Postal 66, CEP - \\ 75.901-970, Rio Verde, Goiás, Brasil.E-mail: cibele.minafra@ifgoiano.edu.br \\ *Autor para correspondência
}

\begin{abstract}
RESUMO. Cada vez mais, consumidores têm demostrado interesse em investir em produtos que garantam o bem-estar dos animais. O Brasil se destaca no assunto avicultura de corte, no entanto a indústria do frango vivencia diariamente com perdas na qualidade do produto final devido ao estresse do manejo pré-abate. Há necessidade de as agroindústrias implantarem programas de bem-estar adequados, que se tornem uma ferramenta essencial para contribuir com melhorias no ambiente de criação e abate, e o mais importante, que não cause sofrimento desnecessário aos animais. Diante deste cenário, é de grande relevância a busca de informações práticas com embasamento científico, aplicáveis a realidade brasileira, que possibilitem reduzir as perdas na qualidade do produto, diminuindo a ocorrência de hematomas, contusões e lesões nas aves e que se preocupem com o bem-estar dos animais. Esta revisão foi realizada para apresentar os principais aspectos do abate humanitário das aves, abordando os padrões do manejo pré-abate e abate, enfatizando as perdas produtivas ocorridas nestas etapas, bem como propor uma reflexão a respeito da ética e bem-estar dos animais.
\end{abstract}

Palavras chave: pré-abate, bem-estar, estresse dos frangos de corte

\section{Humane slaughter of poultry: Review}

\begin{abstract}
Increasingly, consumers have demonstrated interest in investing in products that ensure the welfare of animals. Brazil stands out in the poultry production, however the industry of broilers experiences daily with losses in the quality of the final product due to the stress of pre-slaughter management. There is necessity of agribusiness to implement of an appropriate welfare program, that becomes an essential tool to contribute to improvements in the design environment and the slaughter and most importantly, it does not cause unnecessary suffering to animals. Faced with this scenario, is of great relevance the search of practical information with scientific basis, applicable to Brazilian reality, that allow reducing losses in product quality, reducing the occurrence of hematomas, bruises and injuries in the birds and to be concerned about the welfare of animals. This review was made of present the main aspects of the humane slaughter of poultry, addressing the standards of pre-slaughter and slaughter management, emphasizing the production losses incurred in these steps, and to develop a reflection on the ethics and animal welfare.
\end{abstract}

Keywords: pre-slaughter, welfare, stress of broilers 


\section{Introdução}

É crescente a preocupação da sociedade com os métodos de produção e abate dos animais. Estes princípios éticos se sobressaem entre as novas tendências de mercado, em que os consumidores mais exigentes têm demostrado interesse em investir em produtos que garantam o bem-estar animal.

Atualmente, discute-se muito sobre a qualidade ética da carne, atributo que inclui conceitos como bem-estar animal, sustentabilidade e comprometimento com o meio ambiente. Assim, o abate humanitário ganha importância, e passa ser demanda tanto em pesquisas científicas, quanto em indústrias e propriedades rurais, já que estas exigências podem se tornar barreiras comerciais para produtos.

Com o intuito de padronizar as operações no pré-abate e abate dos animais de produção, foi criado o Programa Nacional de Abate Humanitário (Sociedade Mundial de Proteção Animal-WSPA, 2009). Esse programa foi desenvolvido o objetivo de resgatar a sensibilidade das pessoas, enfatizando a importância de se evitar o sofrimento nos animais.

O termo "abate humanitário" é definido como o conjunto de diretrizes técnicas e científicas que garantem o bem-estar dos animais desde a recepção até a operação de sangria (Brasil, 2000).

Desde 1934 já havia no Brasil a preocupação com o bem-estar animal, sendo estabelecidas medidas de proteção e respeito aos animais pelo Decreto $\mathrm{n}^{\circ} 24.645$ (Brasil, 1934). A Instrução Normativa $n^{\circ} 3 / 2000$ (Brasil, 2000), foi criada para regulamentar os métodos de insensibilização para o abate humanitário de animais de açougue e a Instrução Normativa $\mathrm{n}^{\circ} 56$ (Brasil, 2008), estabelece os procedimentos gerais de recomendações de Boas Práticas de Bem-estar para Animais de Produção e de Interesse Econômico. No entanto, nenhuma dessas leis normatizam todas as fases pré-abate das aves.

Um conjunto de leis dificilmente poderia influir satisfatoriamente sobre ética e bem-estar. Porém, é necessária a formulação de uma lei nacional, completa, específica e criteriosa, que estabeleça parâmetros específicos de conforto dos animais e expresse normas que abranjam todas as etapas do abate humanitário, determinado condutas e padrões de comportamento humano.
Portanto, objetivou-se com este trabalho apresentar os principais aspectos do abate humanitário das aves, abordando os padrões do manejo pré-abate e abate, enfatizando as perdas produtivas ocorridas nestas etapas, bem como propor uma reflexão a respeito da ética e bemestar dos animais.

\section{Bem-estar animal}

Bem-estar animal tem várias definições, mas existe um crescimento consenso de que qualquer que seja a definição, deve incluir três elementos: o estado emocional do animal, o funcionamento biológico e a capacidade de mostrar os padrões normais de comportamento (Manteca et al., 2009).

Estudos etológicos de cognição, motivação e complexidade do comportamento social nos animais levaram a um rápido desenvolvimento da ciência do bem-estar animal. Lida com a saúde, envolve a fisiologia, comportamento, sentimentos e resposta patológica. Nos últimos anos, bemestar animal tem sido estabelecido como um dos critérios utilizados para decidir se um sistema é sustentável e se a qualidade do produto é confiável (Broom \& Molento, 2004).

Para auxiliar na identificação dos principais problemas de bem-estar dos animais, o Conselho de Bem-estar de Animais de Produção - Farm Animal Welfare Council (FAWC, 2009) criou as 5 liberdades dos animais, que representam estados ideais. Um animal é considerado em bom estado de bem-estar se estiver saudável, confortável, bem nutrido, seguro, capaz de expressar seu comportamento inato/natural, e se não estiver sofrendo com dores, medo $\mathrm{e}$ angústias. Bem-estar animal requer prevenção contra doenças e tratamento veterinário, abrigo adequado, gerenciamento, nutrição, manejo cuidadoso e abate humanitário (Bonamigo et al., 2012; Hötzel \& Machado Filho, 2004).

A economia afeta todos os aspectos do debate sobre bem-estar de animais de produção. Investimentos em técnicas que promovem conforto dos animais também aumentam os custos, pelo menos ao curto prazo (Harvey \& Hubbard, 2013). E em países em desenvolvimento, a questão de quem vai arcar com os custos de uma melhor qualidade de vida aos animais de produção é em parte responsável por uma limitação de progressos nessa área (Molento, 2005). 
Bonamigo et al. (2012) e Queiroz et al. (2014) relataram em pesquisas com entrevistados no Brasil, que a maioria dos consumidores não possuem conhecimento suficiente sobre as questões relacionadas ao bem-estar dos animais. Porém, acreditam que uma criação diferenciada pode resultar em melhorias no produto final. $\mathrm{O}$ preço da carne, ainda foi o atributo de maior importância para o consumidor na hora da compra. Mas quando uma reflexão sobre o bemestar animal foi estimulada, a maioria dos consumidores mostraram-se dispostos a pagar um valor adicional pelos produtos com certificação e que garanta maior qualidade.

A interação inadequada do homem com os animais pode aumentar o estresse psicológico e físico, afetando o bem-estar durante as etapas que antecedem o abate. É necessária a implantação de um programa de bem-estar no manejo pré-abate, com objetivo de minimizar sofrimento desnecessário (Ludtke et al., 2010).

Entre os princípios básicos que devem ser observados para atender o bem-estar nas etapas pré-abate estão: métodos de manejo e instalações que reduzam o estresse dos frangos; equipe treinada e capacitada; equipamentos apropriados e método de insensibilização eficaz, que induza a imediata perda de consciência e sensibilidade, para que não cause dor no momento da sangria (Ludtke et al., 2010).

\section{Apanha}

As falhas cometidas nos períodos de précarregamento e carregamento em frangos de corte estão entre as maiores causas de condenações em abatedouros. As lesões observadas nas aves após um carregamento não apropriado podem evoluir de um simples arranhão na pele, a problemas maiores, como contusões, fraturas e edemas. Estes fatores depreciam as carcaças, acarretando prejuízos ao produtor e à agroindústria (Rosa et al., 2013).

O manejo de captura ou apanha é uma importante etapa pré-abate, pois é um dos momentos em que as aves estão mais susceptíveis ao estresse, influenciando diretamente o bemestar e qualidade da carcaça. No Brasil, é realizada a captura manualmente e a apanha, de um modo geral, é realizada por uma equipe de 12 a 14 pessoas (Leandro et al., 2006).

Nijdam et al. (2004) consideraram que a apanha e o transporte durante o dia consistem em um fator de risco. Os autores encontraram percentuais de contusões maiores no período diurno e sugeriram ser resultado da elevada atividade dos frangos em resposta à quantidade de luz. Na apanha noturna, há vantagens de se controlar os níveis de luz dentro dos aviários e os efeitos do calor podem ser minimizados.

O método mecânico de captura de aves tem alto custo e dificuldades na higienização, comprometendo a biossegurança. Nijdam et al. (2005) investigaram os dois métodos de captura: a mecânica e a manual e a influência na mortalidade. Os resultados revelaram altos níveis de corticosterona no plasma sanguíneo das aves estudadas, indicando que ambos os métodos de captura se equivalem à indução de estresse. Em relação à mortalidade, os autores verificaram que a apanha mecânica tende a causar índices superiores.

Pilecco et al. (2011) observaram que quando os pesos de aves/caixa ultrapassaram $30 \mathrm{~kg}$, foram constatados maiores índices de arranhões de pele e, que nas apanhas mais rápidas, havia movimentação brusca e gritos dos membros das equipes, resultando em agitação das aves e consequente aglomeração.

O protocolo de bem-estar para frangos e perus (União Brasileira de Avicultura - UBA, 2008) recomenda que não seja realizada a apanha das aves pelos pés, asas e pescoço devido às lesões e sofrimentos que possam ser causados. Aves com menos de $1,8 \mathrm{~kg}$ podem, excepcionalmente, ser apanhadas pelas pernas, desde que o número máximo em cada mão não seja maior que três. Somente é permitida a apanha pelo dorso de no máximo duas aves por vez.

Leandro et al. (2006) avaliaram a influência do tipo de captura de frangos de corte sobre qualidade na carcaça e notaram que aves capturadas pelo dorso apresentaram menor número de contusões, ou seja, menos condenações no abatedouro do que aquelas que foram pegas pelo pescoço. A captura pelo pescoço aumentou às contusões de carcaça em $33 \%$ e em $72 \%$ as fraturas hemorrágicas.

Adotar práticas de manejo que objetivam o bem-estar, incluindo técnicas que auxiliam na distribuição uniforme das aves, constitui-se na principal ferramenta para evitar arranhões dorsais no momento da apanha. Assim, a organização e capacitação das equipes de apanha e o posicionamento das caixas de forma que subdivida os lotes nos galpões, para facilitar a 
contenção das aves, são manejos que podem amenizar o estresse em frangos de corte (Pilecco et al., 2011).

A idade associada ao peso são os pontos básicos para definição do número de aves por caixa no momento da apanha. Em geral existe pressão para aumento do número de frangos por caixa, buscando redução nos custos com o transporte. Uma recomendação é trabalhar na faixa de 21 a $23 \mathrm{~kg}$ de peso por caixa, mas devese observar o turno de carregamento $e$ as temperaturas de transporte, baseando-se no princípio de que todas as aves devem ter espaço suficiente na caixa para que possam deitar sem ocorrer amontoamento de uma ave sobre a outra (Rosa et al., 2013).

Vosmerova et al. (2010) avaliaram o efeito da apanha e transporte sobre os parâmetros bioquímicos em frangos de corte e observaram maiores níveis de corticosterona e da atividade enzimática da lactato desidrogenase (LDH) antes do transporte, indicando que as etapas prétransporte (apanha e carregamento) podem ser ainda mais estressantes para o frango.

\section{Transporte}

O transporte de aves envolve um dos maiores deslocamentos de animais vivos do mundo. Esta etapa pode ser realizada em diferentes condições e combinações de distâncias, horários e tipo de vias. Estas combinações têm um reflexo direto na qualidade do produto final, e na maioria das vezes serão responsáveis pelas chamadas "mortes na chegada do abatedouro" ou "Dead on Arrivals" (DOA's) (Barbosa Filho et al., 2009).

Os níveis reais de mortalidade dependem de inúmeras variáveis, podendo ser influenciadas pelos seguintes fatores: a saúde dos animais, o estresse térmico e as injúrias e traumas ocorridos nas etapas anteriores ao transporte. É proposto que os desafios térmicos constituem a principal ameaça ao bem-estar das aves e sua sobrevivência. Os efeitos do estresse térmico tendem a aumentar pela retirada prolongada de alimentos e de água e pela exposição à vibrações e acelerações no transporte (Mitchell \& Kettlewell, 2009).

A limitada capacidade de frangos de corte em lidar com o estresse de calor têm resultado em taxas de mortalidade que excedem $1 \%$ durante os dias mais quentes. A alta incidência de lesões e estresse térmico durante o transporte está associada ao mal-estar e representa uma perda considerável para a indústria do frango, especialmente nos trópicos (Vieira et al., 2011).

Em trabalhos com frangos de corte, Chauvin et al. (2011) verificaram que diferentes condições climáticas durante o processo de pré-abate, como a chuva e o vento influenciaram na mortalidade das aves, pois afetaram o conforto térmico durante o transporte ou período de espera no abatedouro.

Silva et al. (2007) realizaram pesquisa para avaliar o efeito da temperatura e umidade relativa do ar (UR) elevadas sobre os parâmetros fisiológicos, numa condição de transporte simulada em câmara climática. A temperatura retal, a frequência respiratória e o hematócrito foram utilizados como parâmetros de medida para estresse fisiológico. Os autores constataram que as aves tenderam a apresentar maiores valores de temperatura retal e frequência respiratória com o aumento do tempo de exposição ao estresse térmico, sendo que, na faixa de condição superior ao estresse, por volta de $46,3^{\circ} \mathrm{C}$, foi registrado óbito.

Vieira et al. (2011) relacionaram o número de mortes encontradas na chegada do abatedouro com a densidade de aves nas caixas, assim como, os turnos em que estas eram transportadas. Os autores verificaram que as densidades elevadas (acima de sete aves por caixa), houve um acréscimo na mortalidade no turno da tarde, seguido do turno da manhã e noite. E nas densidades menores (abaixo de sete aves por caixa), a mortalidade foi elevada no turno da noite, subsequente pelo turno da tarde e pelo turno da manhã. A alta mortalidade no período noturno justificou-se ao efeito da perda de calor sensível.

Barbosa Filho et al. (2009) conduziram ensaio para caracterizar o perfil microclimático da carga de caminhões de transporte de frangos de corte, e observaram que no turno da manhã, as condições ambientais são propícias para esta operação préabate. A região de pior condição microclimática situa-se nas partes central e traseira da carga, o que as colocam como localidades mais suscetíveis de ocorrência de perdas durante o transporte das aves.

O microambiente térmico formado no caminhão pode ser a causa primária que compromete o bem-estar das aves e a qualidade final da carne, com o desenvolvimento de filés de 
peito de frango PSE (Pale, Soft, Exudative) (Simões et al., 2009).

Segundo Warriss et al. (2005), temperaturas de até $17^{\circ} \mathrm{C}$ tem pouco ou nenhum efeito sobre a mortalidade de frangos de corte no transporte pré-abate, sugerindo que o transporte seja realizado em horas mais frescas do dia, como início da manhã, para melhorar os efeitos prejudiciais do clima e contribuir com o bemestar das aves.

A distância entre a granja e o abatedouro também é de grande relevância em consideração às perdas pré-abate, já que ela determina a duração do transporte e o tempo em que as aves ficarão expostas aos efeitos térmicos do ambiente externo. Barbosa Filho et al. (2009) verificaram em sua pesquisa que, quanto maior a distância percorrida no transporte, maior o número de aves mortas na chegada ao abatedouro.

\section{Ambiente da área de espera}

A importância da espera se resume em oferecer, dentro de um espaço de tempo adequado, condições térmicas satisfatórias para manter o animal em conforto após o transporte até o descarregamento. Os frangos devem ser imediatamente colocados na área de espera ambientalmente controlada na chegada às instalações do abatedouro (Humane Farm Animal Care - HFAC, 2008).

Para proporcionar melhores condições, as instalações da área de espera devem ser cobertas e possuir sistemas de aspersão com água e ventiladores e/ou exaustores, posicionados de forma que atinja toda a carga. Nesta área deve haver termo - higrômetro e recomenda-se que a UR do ambiente não ultrapasse $65 \%$ durante o verão (FAWC, 2009).

À medida em que o tempo de transporte e tempo de espera no abatedouro aumentam, há risco de morte das aves. Assim, reduzir percentual DOA e garantir o bem-estar dos frangos de corte durante o último dia de vida é necessário melhorar a logística e planejamento, adequando o tempo de transporte para no máximo de duas horas e manter o período de espera no abatedouro o mais curto possível (Nijdam et al., 2004).

Nääs et al. (1998) analisaram o ambiente de espera dos caminhões de maneira simulada, propondo uma forma de amenizar o estresse térmico com o uso de ventilação e nebulização.
Foi verificada eficiência do sistema, onde as gotículas de água lançadas pelos nebulizadores atinjam as aves e com a ação do vento evaporam, retirando o calor excessivo, proporcionando-lhes uma melhor sensação térmica.

Aves sob condição de estresse térmico não devem ser expostas a longos períodos de espera, pois esse fator não apenas eleva a mortalidade, mas também pode causar danos à carcaça. É importante que as aves permaneçam nos galpões de espera o tempo mínimo necessário para garantir o fluxo de abate e seu bem-estar, sendo recomendado como ideal um período de espera de uma hora, não mais que duas horas. As aves que esperam muito tempo no caminhão podem sofrer problemas de desidratação, já que se encontram sem acesso à água e ração (Ludtke et al., 2010).

Porém, com relação ao tempo de espera nos abatedouros, os resultados ainda são conflitantes. Vieira et al. (2011) verificaram que houve uma redução na mortalidade de frangos no pré-abate, quando o tempo de espera em galpões climatizados foi aumentado. Concluindo assim, que o tempo de espera de 3 a 4 horas num ambiente controlado durante o verão e primavera, foi necessário para reduzir a carga térmica de frangos de corte.

Vieira et al. (2010) avaliaram os efeitos do tempo de espera no bem-estar e mortalidade de frangos transportados em distâncias diferentes granja-abatedouro. Os autores evidenciaram que nas distâncias acima de $25 \mathrm{~km}$, a mortalidade dos frangos foi menor quando adotado o tempo de espera curto. No entanto, no mesmo intervalo de tempo para uma curta distância $(<25 \mathrm{~km})$, o número de aves mortas duplicou em relação à primeira situação.

Como foi exposto, para as curtas distâncias, o tempo de espera em galpão climatizado resultou no retorno à condição inicial de conforto térmico, ou seja, a tensão térmica dos frangos pôde ser reversível, porque eles ainda eram sensíveis ao ambiente climatizado. Para viagens mais longas, as aves ultrapassaram este período crítico, chegando à irreversibilidade devido ao esgotamento das reservas energéticas, sendo menor o efeito da climatização no retorno para o conforto térmico inicial de frangos de corte.

Em estudo, Zhang et al. (2009) verificaram que frangos de corte que tiveram tempo maior de espera no abatedouro após o transporte a longo 
prazo, diminuíram concentrações de corticosterona no plasma e reduziram a glicólise muscular, o que pode ajudar a manter a qualidade da carne.

\section{Pendura}

A etapa de pendura é potencialmente dolorosa para a ave. Na superfície lateral da perna da ave (osso tarso metatarso ou osso da canela) há, aproximadamente de 21 nociceptores, sendo que à medida que aumenta a pressão aplicada nesta região, maior será o número de receptores estimulados enviando sinais de dor. Considera-se ainda, que a uniformidade dos lotes (tamanho e peso) é essencial para evitar problemas de bemestar na linha de abate (Ludtke et al., 2010).

Aves que apresentam fraturas ou lesões que comprometem seu bem-estar, não devem sofrer a pendura. É aceitável o deslocamento manual do pescoço desde que as aves não pesem mais de 3 $\mathrm{kg}$ e que seja realizado por um funcionário treinado para o abate emergencial (FAWC, 2009).

Nesta etapa as aves são manejadas individualmente, exigindo equipe treinada $\mathrm{e}$ número suficiente de colaboradores, para facilitar os cuidados com o animal. Devem ser adotadas medidas apropriadas para evitarem o batimento de asas e tentativa da ave em endireitar-se, que é um reflexo do comportamento de fuga (FAWC, $\underline{2009)}$.

$\mathrm{O}$ apoio para o peito reduz significativamente o número de aves com batimento de asas, devido à segurança causada pelo contato direto com a barra. Os níveis baixos de iluminação na área de pendura e linhas lineares sem obstáculos também têm contribuído para reduzir o estresse nas aves neste período (Ludtke et al., 2010).

O tempo entre pendura e insensibilização deve ser o menor possível, a fim de evitar desconforto nas aves, devido à pressão das pernas com o gancho e a posição invertida em que a aves se encontram. Recomenda-se o tempo máximo de 90 segundos (FAWC, 2009).

O pré-choque é um ponto crítico e deve ser evitado por meio do isolamento elétrico e angulação da rampa de entrada. O pré-choque ocorre quando as aves encostam seu bico ou asas na prancha, antes do mergulho, na cuba de corrente elétrica. Isso causa dor e medo à ave, que se debate e se recolhe, provocando eventuais fraturas, insensibilização inadequada, além do próprio sofrimento (Raj, 1998).

\section{Métodos de insensibilização}

O método mais comum de insensibilização de aves é o elétrico ou eletronarcose em cubas de imersão. O objetivo desta etapa é oferecer às aves um estado de inconsciência no qual há danos temporários da função cerebral e o indivíduo é incapaz de responder aos estímulos normais, incluindo a dor (Erasmus et al., 2010).

Os sistemas de insensibilização elétrica imobilizam a ave tempo suficiente para permitir o alinhamento necessário para a etapa de sangria automatizada, reduzindo danos na carcaça, devido à atividade física inconsciente $(\mathrm{McNeal}$ et al., 2003).

Reflexos de interesse na avaliação da insensibilidade, após o atordoamento, incluem perda de reflexos do tronco cerebral, tais como: o palpebral, córneo e pupilar. Assim como, os reflexos espinais e ausência de respiração rítmica (Erasmus et al., 2010).

Os tanques de imersão devem ser adequados em tamanho e profundidade para o tipo de ave abatida, sua altura deve ser ajustável para permitir que até a base das asas da ave seja imersa. O elétrodo de imersão deve estender a todo o comprimento do tanque. A corrente mínima para atordoamento de aves, quando se utiliza altas frequências $(50 \mathrm{a} 200 \mathrm{~Hz}$ ), é de 100 $\mathrm{mA}$ durante pelo menos 4 segundos (Ludtke et al., 2010).

Para melhorar o fluxo da corrente na cuba e diminuir a resistência, deve-se molhar os ganchos antes que as aves sejam penduradas. Há também a recomendação de não pendurar aves por uma única perna e manter uniformidade do lote para facilitar o contato das pernas com o gancho. Com o mesmo objetivo de melhorar o fluxo da corrente, pode ser adicionado sal $(\mathrm{NaCl})$ na cuba de imersão, já que a condutividade elétrica da água varia conforme sua composição mineral (Ludtke et al., 2010).

As aves podem ser insensibilizadas por exposição a misturas de gases anóxicos, como o gás carbônico e o argônio. As misturas de gases permitidos são projetadas para induzir a perda de consciência sem angústia indevida. Aves podem, em alguns sistemas, ser colocadas na câmara de gás, enquanto que ainda nas caixas de transporte. Outra vantagem sobre o atordoamento elétrico é 
que as aves não sofrem pré-choque e diminuem os problemas com qualidade da carcaça (Ludtke et al., 2010).

\section{Sangria}

A operação de sangria deve ser iniciada logo após a insensibilização do animal, de modo a provocar um rápido, profuso e mais completo possível escoamento do sangue, antes que o animal recupere a sensibilidade (Brasil, 2000).

Uma sangria adequada deve ser realizada seccionando as artérias carótidas e veias jugulares, causando choque hipovolêmico e morte na ave. Após a insensibilização, as aves devem ser sangradas em um tempo máximo de 10 segundos quando utilizadas altas frequências e 20 segundos para uso de baixa frequência (Ludtke et al., 2010). A duração da sangria deve ser, em média de 3 minutos, sendo um processo passivo podendo ser acelerada pelo bombeamento cardíaco. Nos primeiros $40 \%$ do tempo desse processo, os animais devem ter perdido $80 \%$ do sangue (Sarcinelli et al., 2009). No caso de métodos automáticos de sangria, um sistema de back-up manual deve garantir que todas as aves sejam abatidas imediatamente, antes de entrar no tanque de escaldagem (Ludtke et al., 2010).

A sangria realizada de forma incorreta pode causar problemas graves de bem-estar na ave, condenação e depreciação na carcaça. Quando as aves são sangradas de modo ineficiente, ou quando nem passam pela etapa sangria, poderão ser classificadas como uma tecnopatia, em que a carcaça se apresenta com coloração avermelhada e aspecto repugnante (Ludtke et al., 2010).

\section{Considerações finais}

Faz-se necessário a implantação de programas de bem-estar durante o pré-abate e abate das aves, pois a adoção de boas práticas de manejo irá contribuir para qualidade de carcaça final, gerar menores impactos econômicos na cadeia produtiva e garantir um tratamento humanitário às aves. É importante que se aplique as informações técnicas reveladas por pesquisas brasileiras e internacionais na forma de leis, adequando as legislações já existentes ou criando novas normas que padronizem todas as etapas do pré-abate das aves.

\section{Referências Bibliográficas}

Barbosa Filho, J. A. D., Frederico Márcio Corrêa Vieira, F. M. C., Silva, I. J. O., Garcia, D. B., Silva, M. A. N. \& Fonseca, B. H. F. (2009). Transporte de frangos: caracterização do microclima na carga durante o inverno. Revista Brasileira de Zootecnia, 38, 24422446.

Bonamigo, A., Bonamigo, C. B. S. S. \& Molento, C. F. M. (2012). Atribuições da carne de frango relevantes ao consumidor: foco no bem-estar animal. Revista Brasileira de Zootecnia, 41, 1044-1050.

Brasil. Decreto-Lei $\mathrm{n}^{\circ}$ 24.645, de 10 de julho de (1934). Dispõe sobre a proteção animal. Diário Oficial da República Federativa do Brasil. Rio de Janeiro, Suplemento $\mathrm{n}^{\circ} 162$, publicado em 14 de julho de 1934.

Brasil. (2000). Ministério da Agricultura, Pecuária e Abastecimento. Instrução Normativa $\mathrm{N}^{\circ} 3$, de 17 de janeiro de 2000, aprova o regulamento técnico de métodos de insensibilização para abate humanitário de animais de açougue. Diário Oficial da União. Brasília, DF, 24 jan. 2000. Seção 1, p.14.

Brasil. Ministério da Agricultura, Pecuária e Abastecimento. Instrução Normativa $\mathrm{N}^{\circ} 56$, de 06 de novembro (2008), estabelece os procedimentos gerais de recomendação de boas-práticas de bem-estar para animais de produção e de interesse econômico REBEM, abrangendo sistemas de produção e transporte. Diário Oficial da União. Brasília, DF, 07 de nov. de 2008. Seção 1, p.5.

Broom, D. M. \& Molento, C. F. M. (2004). Animal welfare: concept and related issuesreview. Archives of Veterinary Science, 9, 111.

Chauvin, C., Hillion, S., Balaine, L., Michel, V.; Peraste, J., Petetin, I., Lupo, C. \& Le Bouquin, S. (2011). Factors associated with mortality of broilers during transport to slaughterhouse. Animal, 5, 287-293.

Erasmus, M. A., Turner, P. V. \& Widowski. T. M. (2010). Measures of insensibility used to determine effective stunning and killing of poultry. The Journal of Applied Poultry Research, 19, 288-298.

FAWC. (2009). Farm animal welfare in Great Britain: Past, present and future. Farm Animal Welfare Council, England. 
Harvey, D. \& Hubbard, C. (2013). Reconsidering the political economy of farm animal welfare: An anatomy of market failure. Food Policy 38: 105-114.

Hötzel, M. J., \& Machado Filho, L. C. P. (2004). Bem-estar animal na agricultura do século XXI. Revista de Etologia, 6, 3-15.

Leandro, N. S. M., Rocha, P. T., Stringhini, J. H., Schaitl, M. \& Fortes, R. M. (2006). Efeito do tipo de captura dos frangos de corte sobre a qualidade da carcaça. Ciência Animal Brasileira, 2, 97-100.

Ludtke, C. B., Ciocca, J. R. P., Dandin, T., Barbalho, P. C. \& Vilela, J. A. (2010). Abate humanitário de aves. WSPA Brasil, Rio de Janeiro.

Manteca, X., Velarde, A. \& Jones, B. 2009. Animal welfare components. In: F. Smulders \& B. Algers (eds.) Welfare of production animals: assessment and management of risks. p 61-77, Wageningen.

McNeal, W. D., Fletcher, D. L. \& Buhr, R. J. 2003. Effects of stunning and decapitation on broiler activity during bleeding, blood loss, carcass, and breast meat quality. Poultry Science, 82, 163-168.

Mitchell, M. A. \& Kettlewell, P. J. 2009. Welfare of poultry during transport-a review. In: Poultry Welfare Symposium, 90-100.

Molento, C. F. M. 2005. Bem-estar e produção animal: Aspectos econômicos - Revisão. Archives of Veterinary Science, 10, 1-11.

Nääs, I. A., Silva, I. J. O. \& Gouveia, R. P. 1998. Avaliação de sistemas de resfriamento no ambiente de espera em caixas transportadoras de frango de corte utilizando a ventilação e nebulização. Revista de Engenharia Rural, 9: 50-55.

Nijdam, E., Arens, P. Lambooij, E. Decuypere, E. \& Stegeman, J. A. 2004. Factors influencing bruises and mortality of broilers during catching, transport, and lairage. Poultry Scice, 83 1610-1615.

Nijdam, E., Delezie, E., Lambooij, E., Nabuurs, M. J. A., Decuypere, E. \& Stegeman, J. A. 2005. Comparison of bruises and mortality, stress parameters, and meat quality in manually and mechanically caught broilers. Poultry Scice, 84, 467-474.
Pilecco, M., Paz, I. C. L. A., Tabaldi, L. A., Nääs, I. A., Garcia, R. G., Caldara, F. R. \& Cavichiolo, F. 2011. Influência de fatores genéticos, ambientais e de manejo sobre a incidência de arranhões dorsais em frangos de corte. Revista Agrarian 4: 352-358.

Queiroz, M. L. V., Barbosa Filho J. A. D., Albiero, D., Freitas Brasil, D. \& Melo. R. P. (2014). Percepção dos consumidores sobre o bem-estar dos animais de produção em Fortaleza, Ceará. Revista Ciência Agronômica, 45, 379-386.

Raj, M. (1998). Welfare during stunning and slaughter of poultry. Poultry Science, 77, 1815-1819.

Rosa, P. S., Albino, J. J., Bassi, L. J., Grah, R. A. \& Niendiccker, T. P. (2013). Manejo préabate em frangos de corte. Embrapa Suínos e Aves-Recomendação Técnica 1, 1-2.

Sarcinelli, M. F., Venturini, K. S. \& Silva, L. C. S. (2009). Abate de aves. Boletim técnicoPIE-UFES, 607, 1-7.

Silva, M. A. N., Barbosa Filho, J. A. D., Silva, C. J. M., Rosário, M. F., Silva, I. J. O., Coelho, A. A. D. \& Savino, V. J. M. (2007). Avaliação do estresse térmico em condição simulada de transporte de frangos de corte. Revista Brasileira de Zootecnia, 36, 11261130.

Simões, G. S., Oba, O.; Matsuo, T., Rossa, A., Shimokomaki, A. \& Ida, E. L. (2009). Vehicle thermal microclimate evaluation during Brazilian summer broiler transport and the occurrence of PSE (pale, soft, exudative) meat. Meat. Brazilian Archives of Biology and Technology, 52, 195-204.

WSPA, Sociedade Mundial de Proteção Animal. Abate Humanitário. (2009). Disponível em: <http://www.wspabrasil.org/wspaswork/factor yfarming/Abatehumanitario.aspx $>$. Acesso em: 18 abr. 2015.

Vieira, F. M. C., Silva, I. J. O., Barbosa Filho, J. A. D. \& Vieira, A. M. C. (2010). Productive losses on broiler preslaughter operations: effects of the distance from farms to abattoirs and of lairage time in a climatized holding area. Revista Brasileira de Zootecnia, 39, 2471-2476.

Vieira, F. M. C., Silva, I. J. O., Barbosa Filho, J. A. D., Vieira, A. M. C. \& Broom, D. M. (2011). Preslaughter mortality of broilers in 
relation to lairage and season in a subtropical climate. Poultry Science, 90, 2127-2133.

Vosmerova, P., Chloupek, J., Bedanova, I., Chloupek, P., Kruzikova, K., Blahova, J. \& Vecerek, P. (2010). Changes in selected biochemical indices related to transport of broilers to slaughterhouse under different ambient temperatures. Poultry Science, 89, 2719-2725.

Warriss, P. D., Pagazaurtundua, A. \& Brown, S. N. (2005). Relationship between maximum daily temperature and mortality of broiler chickens during transport and lairage. Poultry Science, 46: 647-651.
Zhang, L, Yue, H. Y., Zhang, H. J., Xu, L., Wu, S. G., Yan, H. J., Gong, Y. S. \& Qi, G. H. (2009). Transport stress in broilers: I. Blood metabolism, glycolytic potential, and meat quality. Poultry Science, 88, 2033-2041.

\section{Article History}

Received 27 June 2016

Accepted 12 July 2016

Available on line 29 July 2016

License information: This is an open-access article distributed under the terms of the Creative Commons Attribution License, which permits unrestricted use, distribution, and reproduction in any medium, provided the original work is properly cited. 